\title{
Fosfomycin Susceptibility in Multidrug Resistant Urinary Escherichia coli Isolates
}

\author{
Greeshma Hareendranath ${ }^{1}$, Aiswarya Mukundan², Sreekumary Puthuseril Kunjappan³ \\ ${ }^{1}$ Department of Microbiology, Government T.D. Medical College, Alappuzha, Kerala, India. \\ ${ }^{2}$ Department of Microbiology, Government Medical College, Thrissur, Kerala, India. \\ ${ }^{3}$ Department of Microbiology, Government Medical College, Kottayam, Kerala, India.
}

\section{ABSTRACT}

\section{BACKGROUND}

Escherichia coli is one of the most important causes of urinary tract infections (UTIs). Increased antibiotic resistance may limit the therapeutic options for the treatment of these infections. Fosfomycin trometamol is a phosphonic acid derivative, which acts primarily by interfering with bacterial peptidoglycan synthesis with broad spectrum of activity against agents causing urinary tract infection with good antibiofilm activity and limited reports of resistance and hence is increasingly called upon for the treatment of multi drug resistant (MDR) organisms causing UTI. There are limited studies from India regarding the efficacy of this drug; so, the study was conducted to determine the in vitro efficacy of fosfomycin against uropathogenic MDR E. coli.

\section{METHODS}

This was a prospective study done in the Department of Microbiology, Government T.D. Medical College, Alappuzha, over a period of 1 year from April 2018 to March 2019. A total of 150 MDR urine samples were processed by routine microbiological methods and after identification of E. coli urinary isolates, antibiotic susceptibility testing was performed and results were interpreted following the Clinical and Laboratory Standards Institute guidelines (CLSI). Fosfomycin sensitivity was tested by the Kirby-Bauer disc diffusion method.

\section{RESULTS}

Among the 150 MDR urinary E. coli isolates, 148 (98\%) were sensitive to fosfomycin in our study. The susceptibility rate of fosfomycin was clearly higher than other commonly used drugs for UTI. All extended-spectrum beta-lactamases (ESBL) producing E. coli were sensitive to this drug. The susceptibility for nitrofurantoin was fair, whereas for ampicillin, norfloxacin, cefotaxime and trimethoprim / sulphamethoxazole was found poor. Relatively better rates of resistance were observed for parenteral antibiotics.

\section{CONCLUSIONS}

With an enormous increase in the bacterial pathogens resistant to first-line antibiotics, there has been a revival in the use of fosfomycin. The convenience of a single dose regimen, a good activity proven invitro, and minimal propensity for development of resistance pathogens makes fosfomycin an attractive regimen for the treatment of uncomplicated community and hospital acquired UTIs. In this regard, with the existing limited options for treating MDR organisms, fosfomycin finds its utility acting as an effective and promising option in the treatment of UTIs due to MDR pathogens in the future.

\section{KEY WORDS}

Urinary Tract Infection, Multidrug Resistant Escherichia coli, Fosfomycin

\section{Corresponding Author:} Dr. Greeshma Hareendranath, Department of Microbiology, Government T.D. Medical College, Alappuzha - 688005, Kerala, India. E-mail: greeshmaanoop@ymail.com

DOI: 10.14260/jemds/2021/92

How to Cite This Article: Hareendranath G, Mukundan A, Kunjappan SP. Fosfomycin susceptibility in multidrug resistant urinary Escherichia coli isolates. J Evolution Med Dent Sci 2021;10(07):414418, DOI: 10.14260/jemds/2021/92

Submission 19-08-2020,

Peer Review 23-11-2020,

Acceptance 30-11-2020,

Published 15-02-2021.

Copyright (C) 2021 Greeshma Hareendranath et al. This is an open access article distributed under Creative Commons Attribution License [Attribution 4.0 International (CC BY 4.0)] 


\section{BACKGROUND}

Urinary tract infections are among the commonest types of bacterial infections. Antimicrobial resistance is increasing worldwide, and this has resulted in infections that are more difficult to treat and are associated with higher mortality, morbidity and cost. The treatment for UTI has posed a challenge to physicians due to the emergence of multidrug resistant uropathogens. Many antibiotics once used to treat UTI are now ineffective due to the development of antimicrobial resistance. Only a few new antibiotics are on the horizon and those that have been recently approved are mostly for intravenous use. The overuse and misuse of antibiotics have led to the emergence of antibiotic resistance in the community making most of the available oral antibiotics ineffective for use. With increasing numbers of patients with uncomplicated urinary tract infections requiring hospitalisation for intravenous antibiotics because of limited oral treatment options, efforts are being made to develop newer antibiotics for outpatient UTI treatment thereby minimising healthcare costs. Fosfomycin, discovered in late 1960 s, has rekindled clinical interests because of reported susceptibilities of current pathogens to the agent. This old 'forgotten' drug is now being re-explored for the treatment of cystitis. It has the unique properties of not sharing any structural similarity and lack of cross-resistance with other antimicrobial agents. ${ }^{1}$ It is a broad-spectrum bactericidal agent that inhibits the MurA enzyme (UDP-Nacetylglucosamine enol pyruvyl transferase) involved in the synthesis of the essential peptidoglycan component, $\mathrm{N}$ acetylmuramic acid of the cell wall. ${ }^{2}$ Fosfomycin is reported to achieve high concentrations in the urinary tract, with a minimal impact on gastrointestinal flora and a low propensity for resistance. Fosfomycin tromethamine has been approved as an oral single-dose treatment for acute uncomplicated cystitis. ${ }^{3}$ In many countries it is now a first-line treatment option for uncomplicated urinary tract infection in women. ${ }^{4}$ According to the guidelines published by the Infectious Diseases Society of America (IDSA) and the European Society for Clinical Microbiology and Infectious Diseases (ESCMID) the recommendations are fosfomycin, nitrofurantoin, and trimethoprim-sulfamethoxazole (TMP-SMX) as first-line agents to treat acute uncomplicated UTIs in adult females, reserving fluoro-quinolones, amoxicillin-clavulanate, and other beta-lactams as second-line agents. 4

The mechanism of resistance to fosfomycin has been associated most commonly with chromosomal mutations. ${ }^{5}$ It is also important to monitor the trends of resistance to fosfomycin in the E. coli causing UTI. The emergence of resistance is a reasonable concern when evaluating fosfomycin for clinical use.

In this study we wanted to evaluate in-vitro susceptibility of fosfomycin among multidrug resistant urinary isolates of Escherichia coli (E. coli).

\section{METHODS}

This is a descriptive study conducted over a period of 1 year from April 2018 to March 2019 in the Department of Microbiology, Government T. D. Medical College, Alappuzha.
The study was approved by the institutional ethical committee.

\section{Study Population}

Urine samples of all patients received in the Department of Microbiology, Govt. T.D. Medical College, Alappuzha, during the study period were analysed. Urinary isolates other than $E$. coli and $E$. coli isolates sensitive to more than three classes of antibiotics were excluded from the study.

\section{Data Collection Procedure}

Urine samples received in the microbiology laboratory from both inpatients and outpatients were processed immediately. Direct microscopy of the uncentrifuged urine sample was done to look for pus cells and bacteria. The samples were plated by semi-quantitative culture method on blood agar and MacConkey`s agar and incubated at $37^{\circ} \mathrm{C}$ overnight. The growth of organisms and colony count was determined. The isolates obtained from the samples with significant bacteriuria in the background of relevant supportive clinical features of UTI and / or the presence of significant pus cells on direct microscopy, as described in the standard guidelines, were proceeded. ${ }^{6}$ The bacterial species identification was performed by standard biochemical methods. ${ }^{7}$ Antimicrobial susceptibility testing was performed on Mueller-Hinton agar plate using Kirby-Bauer disc diffusion method and results were interpreted according to the Clinical and Laboratory Standards Institute guidelines. ${ }^{8}$ The different antimicrobial agents that were tested are ampicillin $(25 \mu \mathrm{g})$, cefotaxime (30 $\mu \mathrm{g})$, ciprofloxacin $(5 \mu \mathrm{g})$, norfloxacin $(10 \mu \mathrm{g})$, gentamicin $(10$ $\mu \mathrm{g})$, cefotaxime $(30 \mu \mathrm{g})$, trimethoprim / sulphamethoxazole $(1.25 / 23.75 \mu \mathrm{g})$, amikacin $(30 \mu \mathrm{g})$, nitrofurantoin $(300 \mu \mathrm{g})$, piperacillin-tazobactam $(100 / 10 \mu \mathrm{g})$, meropenem $(30 \mu \mathrm{g})$. ESBL production in E. coli was tested with cefotaxime $(30 \mu \mathrm{g})$ and combination of cefotaxime / clavulanate $(30 / 10 \mu \mathrm{g})$ by disc diffusion method. ${ }^{8}$

Multi drug resistant pathogens were defined as organisms resistant to three or more agents in different antimicrobial classes according to the standard definition. ${ }^{9}$ All the isolates identified as multidrug resistant were tested with fosfomycin $(200 \mu \mathrm{g})$.

\section{Statistical Analysis}

Data was entered in Microsoft Excel and analysed using SPSS software. Quantitative variables were expressed as percentages.

\section{RESULTS}

In our study, Escherichia coli was the most common Enterobacteriaceae to be isolated. Out of 520 total E. coli isolated some were identified as coliforms and were not included as it could not be satisfactorily included as typical $E$. coli but only as $E$. coli variants. Hence out of 375 typical $E$. coli identified, our study had 150 MDR $E$. coli isolates, majority was from inpatient samples and belonged to the age group $>12$ years. The male to female distribution was almost equal with 
$47 \%$ females and $52 \%$ males. The resistance pattern of $E$. coli to all the antibiotics tested is depicted in [Figure 1].

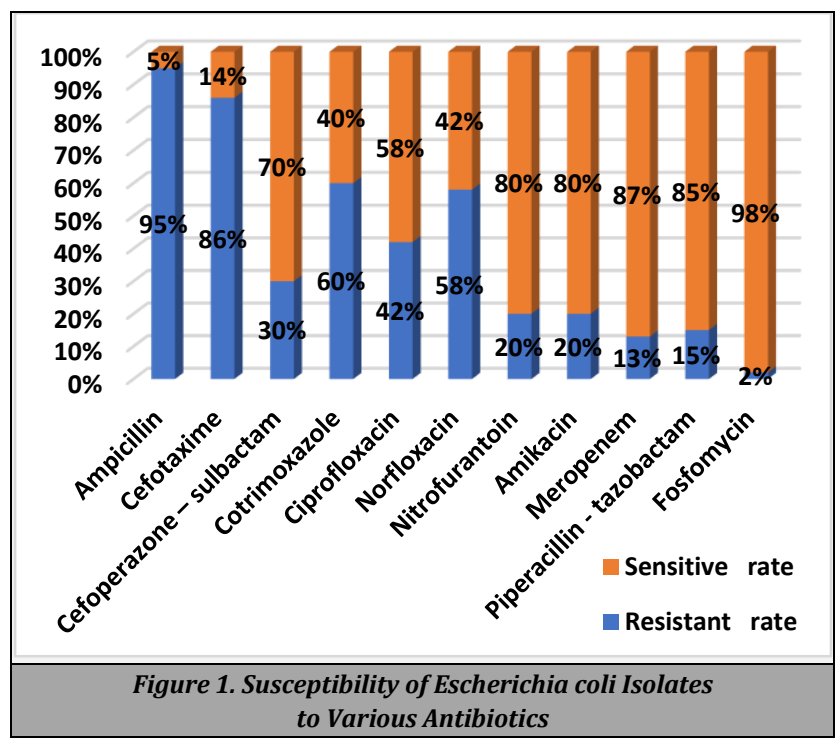

\section{DISCUSSION}

In our study, it can be seen that there is a high amount of resistance to most of the routinely used antimicrobials against uropathogenic E. coli. The commonly used antibiotics like ampicillin, cotrimoxazole and fluoroquinolones are widely resistant which is in accordance to many other researchers as well. ${ }^{10,11,12}$ Various studies from India also support the above finding. ${ }^{13,14}$ There is a considerable increase in resistance to cefotaxime also. About 129 (86 \%) isolates were resistant to cefotaxime and $75 \%$ of these were ESBL producing E. coli. In our study, all the ESBL producing strains showed $100 \%$ sensitivity to fosfomycin. nitrofurantoin, the age-old urinary antibiotic has also shown an effective susceptibility rate in our study. Out of the 150 MDR E. coli isolates, 120 (80\%) were sensitive to nitrofurantoin. The study by Simon A et al and Lai B et al, have also shown a considerable susceptibility rate of $E$. coli isolates to nitrofurantoin. ${ }^{15,16}$

The data that is presented in this study regarding the antibacterial activity of fosfomycin against uropathogenic $E$. coli, support the findings of studies performed worldwide.12-16 We could demonstrate a remarkable antibacterial activity of fosfomycin against MDR E. coli, as high as $98 \%$ of the E. coli isolates were sensitive to fosfomycin. Falagas et al and Maraki et al, have also shown promising results with fosfomycin.17,18 Many clinical studies have shown fosfomycin to be effective for the treatment of lower UTIs due to ESBL producing members of the enterobacteriaceae.19,20,21. It has been reported that fosfomycin has an excellent invitro activity against MDR enterobacteriaceae including both ESBL producing and carbapenem-resistant pathogens particularly uropathogenic E. coli. ${ }^{22,23}$ In the study by Gupta et al, among 150 uropathogenic E. coli, $\mathbf{5 2 . 6 \%}$ of isolates were ESBL producers, and all these strains were susceptible to fosfomycin. ${ }^{23}$ According to Gopichand et al and Batra et al, fosfomycin had the highest in vitro susceptibility followed by nitrofurantoin which is again in accordance with our study. ${ }^{24,25}$ Although the level of resistance to many of the routinely used antibiotics for
UTI is high, in our study it is evident that both nitrofurantoin and fosfomycin have good efficacy against MDR E. coli. According to Rajendran et al, fosfomycin was the only antibiotic that effectively inhibited most strains of MDR $E$. coli. ${ }^{26}$ In another study by Karlowsky et al, they have reported a remarkable susceptibility of fosfomycin against urinary isolates of Escherichia coli, collected from 2010 to 2013 as part of the Canadian national surveillance study CANWARD. ${ }^{27}$ There are also reports of fosfomycin with promising results following treatment of urinary tract infection in clinical practice. ${ }^{28,29,30}$

There is an alarming increase in the resistance rates of urinary E. coli isolates to trimethoprim-sulfamethoxazole and ciprofloxacin and norfloxacin, which are the other antimicrobial agents frequently prescribed for the treatment of cystitis. ${ }^{31,32}$ With the emergence of community-acquired UTIs caused by ESBL-producing MDR E. coli in increasing frequency, often the clinicians are left with only a few oral treatment options. ${ }^{33}$

Due to the emergence of resistance to many regularly used antibiotics, attributable to the irrational use and to the ease of availability of antibiotics over the counter, there is definitely a need for introducing newer drugs that can be used to combat the current situation. Hence, in this scenario, fosfomycin, finds its utility and could be a preferable option for treatment of UTI having demonstrated an excellent in vitro activity against common uropathogens, including MDR isolates.

Fosfomycin currently attracts clinicians' interest worldwide, with the reported high efficiency and activity against pathogens with advanced resistance suggesting that this oral antibiotic could a reliable option for patients with difficult-to-treat-infections. It is commercially available in oral formulation as a single-dose tablet and is best absorbed if given before food intake and is excreted in urine. Data suggests that a single oral dose of fosfomycin trometamol $3 \mathrm{~g}$ (the approved dosage) achieves high concentrations in urine. It achieves a urinary concentration of $2000 \mu \mathrm{g} / \mathrm{mL}$ and maintains high levels for over 24 hours. Hence, single-time oral therapy with fosfomycin has been recommended in uncomplicated UTI. ${ }^{34}$ Fosfomycin has also demonstrated a favourable safety profile, and various clinical trials have demonstrated excellent efficacy in the treatment of uncomplicated cystitis comparable to other first-line antimicrobials. ${ }^{20,21}$ Due to its good renal excretion, fosfomycin gets concentrated in urine which enhances its ability to break up biofilms. The effect of fosfomycin on biofilm was determined by Gopichand et al and they have concluded that fosfomycin could inhibit biofilm formation. ${ }^{24}$ The low resistance to nitrofurantoin and fosfomycin, as evident in our study suggests that these two antibiotics can be considered in the treatment of uncomplicated UTI. Fosfomycin can be considered a preferable option over fluoroquinolones for the treatment of UTI's thereby sparing fluoroquinolones for other systemic infections.

Fosfomycin has a unique chemical structure that is distinct from all other marketed antimicrobial classes ( $\beta$-lactams, glycopeptides, fluoroquinolones, macrolides, lincosamides, tetracyclines, aminoglycosides) and there is no crossresistance with other agents used to treat cystitis. ${ }^{35}$ It has a broad spectrum of activity against aerobic Gram positive and Gram-negative bacteria, including those that cause urinary tract infections. Fosfomycin has good in vitro activity against 
common uropathogens, such as Escherichia coli (including extended-spectrum $\beta$-lactamase-producing E. coli), Proteus mirabilis, Proteus vulgaris, Klebsiella pneumoniae, Pseudomonas aeruginosa, Staphylococcus saprophyticus, and enterococci including vancomycin resistant enterococci (VRE) and the susceptibility of uropathogens to fosfomycin has remained relatively stable over time. ${ }^{36,37}$ Results of recent randomised trials indicate that single-dose fosfomycin trometamol had similar clinical and / or bacteriological efficacy to 3 to 7 day regimens of ciprofloxacin, norfloxacin, cotrimoxazole or nitrofurantoin in women with uncomplicated lower UTIs. ${ }^{3}$

With the purpose of evaluating fosfomycin and also to compare the potential of certain older antibiotics in the treatment of UTIs, especially against MDR E. coli, we could demonstrate that aminoglycosides and carbapenems showed good results, but all these are parenteral antibiotics. Our study could also demonstrate significant resistance to cotrimoxazole and norfloxacin, This is similar to data observed by other authors as well. ${ }^{25}$ The available options for oral antibiotics for the treatment of UTI are limited due to the widespread misuse of these drugs for every outpatient indication and lack of implementation of adequate guidelines for prescribing antibiotics. In our study the notable exceptions are two oral antibiotics, nitrofurantoin and fosfomycin, which have shown an effective susceptibility against MDR $E$. coli which again is in concordance with other researchers as well. ${ }^{11}$

The spread of ESBLs among isolates of Enterobacteriaceae both from community and health-care settings is quite an alarming finding. This often becomes a challenge for the practicing physician in choosing the appropriate antibiotic therapy for treating UTIs caused by MDR pathogens at the appropriate time. While options like carbapenems, tigecycline, $\beta$-lactam / $\beta$-lactamase inhibitor combinations exist, they are flawed by various factors like their unfriendly regimens and parenteral use, thereby negating their role in the outdoor patients. In this regard, there is definitely the need for a newer drug that is orally active, and has low levels of existing resistance and also doesn't encourage antimicrobial resistance in future.

Single-dose fosfomycin trometamol is generally known to be well tolerated, with gastrointestinal adverse events (diarrhoea, nausea) occasionally reported. ${ }^{25}$ Resistance rate of fosfomycin trometamol is low and most frequently acquired by chromosomal mutations that do not spread easily. ${ }^{38}$ It has also been approved in numerous countries worldwide, mainly for the treatment of uncomplicated UTIs. Owing to its unique mechanism of action, broad spectrum of activity, minimal adverse effects and low incidence of resistance, fosfomycin can definitely be considered as a potential therapeutic alternative option for the first-line empirical treatment of uncomplicated lower UTI in the future.

\section{CONCLUSIONS}

Urinary tract infections are one of the commonest primary diagnoses in health-care settings and the emergence of resistance to regularly used antibiotics has resulted in limited therapeutic options. Currently, only a few newer antibiotics are in the pipeline of development. It is in this scenario, that fosfomycin has reclaimed its utility in the treatment of UTI.
Recent interest in fosfomycin tromethamine for treatment of multi-drug-resistant organisms causing UTIs is due to the oral therapy choices being limited. In view of the numerous advantages, fosfomycin is definitely a promising alternative to currently available first-line antibiotics for the treatment of uncomplicated UTI, proven that the resistance to this drug is significantly low. The easy dosage schedule of this drug also ensures compliance thereby discouraging the development of any resistance that may occur because of faulty patient habits.

Fosfomycin is active in vitro against MDR urinary isolates. Fosfomycin may be considered a useful antibiotic agent for both outpatient and inpatient therapy for the treatment of UTIs.

Data sharing statement provided by the authors is available with the full text of this article at jemds.com.

Financial or other competing interests: None.

Disclosure forms provided by the authors are available with the full text of this article at jemds.com.

We would like to thank Dr Anoop Nair, for his constant encouragement and guidance.

\section{REFERENCES}

[1] Michalopoulos AS, Livaditis IG, Gougoutas V. The revival of fosfomycin. Int J Infect Dis 2011;15(11):e732-9.

[2] Brown ED, Vivas EI, Walsh CT, et al. MurA (MurZ), the enzyme that catalyzes the first committed step in peptidoglycan biosynthesis, is essential in Escherichia coli. J Bacteriol 1995;177(14):4194-7.

[3] Keating GM. Fosfomycin trometamol: a review of its use as a single-dose oral treatment for patients with acute lower urinary tract infections and pregnant women with asymptomatic bacteriuria. Drugs 2013;73(17):1951-66.

[4] Gupta K, Hooton TM, Naber KG, et al. International clinical practice guidelines for the treatment of acute uncomplicated cystitis and pyelonephritis in women: a 2010 update by the Infectious Diseases Society of America and the European Society for Microbiology and Infectious Diseases. Clin Infect Dis 2011;52(5):e103-20.

[5] Kobayashi S, Kuzuyama T, Seto H. Characterization of the fomA and fomB gene products from Streptomyces wedmorensis, which confer fosfomycin resistance on Escherichia coli. Antimicrob Agents Chemother 2000;44(3):647-50.

[6] Mandell GL, Bennett JE, Mandell DR. Douglas and Bennett's Principles and practices of infectious diseases. $6^{\text {th }}$ edn. Philadelphia: Elsevier Churchill Livingstone 2005:2789-95.

[7] Collee JG, Fraser AG, Marmion BP, et al. Tests for identification of bacteria. In: Collee JG, Mackie TJ, McCartney JE, ed. Mackie and McCartney practical medical microbiology. 14th edn. London: Churchill Livingstone 1996:131-49.

[8] Clinical and Laboratory Standards Institute: performance standards for antimicrobial susceptibility testing; 27th Informational Supplement, CLSI M100-S27. Wayne PA, USA: Clinical and Laboratory Standards Institute 2017.

[9] Magiorakos AP, Srinivasan A, Carey RB, et al. Multidrugresistant, extensively drug resistant and Pandrug resistant bacteria: an international expert proposal for 
interim standard definitions for acquired resistance. Clin Microbiol Infect 2012;18(3):268-81.

[10] Linsenmeyer K, Strymish J, Weir S, et al. Activity of Fosfomycin against Extended-Spectrum- $\beta$-LactamaseProducing Uropathogens in Patients in the Community and Hospitalized Patients. Antimicrob Agents Chemother 2015;60(2):1134-6.

[11] Anand M, Sahu C, Negi A, et al. In vitro assessment of fosfomycin: a beacon of hope in drug resistant organisms causing urinary tract infections. Journal of Advances in Medicine and Medical Research 2019;30(2):1-9.

[12] Liu HY, Lin HC, Lin YC, et al. Antimicrobial susceptibilities of urinary extended-spectrum beta-lactamase-producing Escherichia coli and Klebsiella pneumoniae to fosfomycin and nitrofurantoin in a teaching hospital in Taiwan. J Microbiol Immunol Infect 2011;44(5):364-8.

[13] Patwardhan V, Singh S. Fosfomycin for the treatment of drug-resistant urinary tract infections: potential of an old drug not explored fully. Int Urol Nephrol 2017;49(9):1637-43.

[14] Sardar A, Basireddy SR, Navaz A, et al. Comparative evaluation of fosfomycin activity with other antimicrobial agents against E.coli isolates from urinary tract infections. J Clin Diagn Res 2017;11(2):DC26-9.

[15] Auer S, Wojna A, Hell M. Oral treatment options for ambulatory patients with urinary tract infections caused by extended-spectrum- $\beta$-lactamase-producing Escherichia coli. Anti-microbial Agents and Chemotherapy 2010;54(9):4006-8.

[16] Lai B, Zheng B, Li Y, et al. In vitro susceptibility of Escherichia coli strains isolated from urine samples obtained in mainland China to fosfomycin trometamol and other antibiotics: a 9-year surveillance study (20042012). BMC Infect Dis 2014;14:66.

[17] Falagas ME, Kanellopoulou MD, Karageorgopoulos DE, et al. Antimicrobial susceptibility of multidrug-resistant Gram negative bacteria to fosfomycin. Eur J Clin Microbiol Infect Dis 2008;27(6):439-43.

[18] Maraki S, Samonis G Rafailidis PI, et al. Susceptibility of urinary tract bacteria to fosfomycin. Antimicrob Agents Chemother 2009;53(10):4508-10.

[19] Pullukcu H, Tasbakan M, Sipahi OR, et al. Fosfomycin in the treatment of extended spectrum beta-lactamaseproducing Escherichia coli-related lower urinary tract infections. Int J Antimicrob Agents 2007;29(1):62-5.

[20] Neuner EA, Sekeres J, Hall GS, et al. Experience with fosfomycin for treatment of urinary tract infections due to multidrug-resistant organisms. Antimicrob Agents Chemother 2012;56(11):5744-8.

[21] Falagas ME, Kastoris AC, Kapaskelis AM, et al. Fosfomycin for the treatment of multidrug-resistant, including extended spectrum beta-lactamase producing, Enterobacteriaceae infections: a systematic review. Lancet Infect Dis 2010;10(1):43-50.

[22] Banerjee S, Sengupta M, Sarker TK. Fosfomycin susceptibility among multidrug resistant, extendedspectrum beta-lactamase-producing, carbapenemresistant uropathogens. Indian J Urol 2017;33(2):149-54.

[23] Gupta V, Rani H, Singla N, et al. Determination of extended-spectrum $ß$-lactamases and AmpC production in uropathogenic isolates of Escherichia coli and susceptibility to fosfomycin. J Lab Physicians 2013;5(2):90-3.

[24] Gopichand P, Agarwal G, Natarajan M, et al. In vitro effect of fosfomycin on multi-drug resistant gram-negative bacteria causing urinary tract infections. Infect Drug Resist 2019;12:2005-13.

[25] Batra P, Abrol AK, Gupta S, et al. Susceptibility pattern of oral antimicrobials in uncomplicated UTI: does fosfomycin still stand effective? J Family Med Prim Care 2020;9(2):850-3.

[26] Rajenderan S, Balaji V, Anandan S, et al. Determination of MIC distribution of arbekacin, cefminox, fosfomycin, biapenem and other antibiotics against gram-negative clinical isolates in South India: a prospective study. PLoS One 2014;9(7):e103253.

[27] Karlowsky JA, Denisuik AJ, Lagacé-Wiens PRS, et al. In vitro activity of fosfomycin against Escherichia coli isolated from patients with urinary tract infections in Canada as part of the CANWARD surveillance study. Antimicrob Agents Chemother 2014;58(2):1252-6.

[28] Seroy JT, Grim SA, Reid GE, et al. Treatment of MDR urinary tract infections with oral fosfomycin: a retrospective analysis. J Antimicrob Chemother 2016;71(9):2563-8.

[29] Matthews PC, Barrett LK, Warren S, et al. Oral fosfomycin for treatment of urinary tract infection: a retrospective cohort study. BMC Infect Dis 2016;16(1):556.

[30] Qiao LD, Zheng B, Chen S, et al. Evaluation of three-dose fosfomycin tromethamine in the treatment of patients with urinary tract infections: an uncontrolled, open-label, multicentre study. BMJ Open 2013;3(12):e004157.

[31] Walkty A, Lagacé-Wiens PRS, Karlowsky JA, et al. Change in antimicrobial susceptibility of Escherichia coli urinary tract isolates at a single institution over a period of 10 years. Can J Microbiol 2012;58(3):345-9.

[32] Karlowsky JA, Lagacé-Wiens PRS, Simner PJ, et al. Antimicrobial resistance in urinary tract pathogens in Canada from 2007 to 2009: CANWARD sur-veillance study. Antimicrob. Agents Chemother 2011;55(7):316975.

[33] Doi Y, Park YS, Rivera JI. et al. Community-associated extended-spectrum $\beta$-lactamase-producing Escherichia coli infection in the United States. Clin Infect Dis 2013;56(5):641-8.

[34] Raz R. Fosfomycin: an old -- new antibiotic. Clin Microbiol Infect 2012;18(1):4-7.

[35] Popovic M, Steinort D, Pillai S, et al. Fosfomycin: an old, new friend? Eur J Clin Microbiol Infect Dis 2010;29(2):127-42.

[36] Greenwood D, Edwards R, Brown J, et al. The comparative activity of fosfomycin trometamol against organisms isolated from infected urines. Infection 1992;20 Suppl 4:S302-4.

[37] Barry AL, Fuchs PC. In vitro susceptibility testing procedures for fosfomycin tromethamine. Antimicrob Agents Chemother 1991;35(6):1235-8.

[38] Karageorgopoulos DE, Wang R, Yu XH, et al. Fosfomycin: evaluation of the published evidence on the emergence of antimicrobial resistance in gram-negative pathogens. J Antimicrob Chemother 2012;67(2):255-68. 